\title{
The incidence of the dumping syndrome following gastrojejunostomy with Roux-en-y anastomosis
}

\author{
RICHARD J. CADE \\ F.R.A.C.S., F.R.C.S.
}

JOHN O. KILBY

M.S., F.R.C.S.

Gloucestershire Royal Hospital, Great Western Road, Gloucester GL1 3NN

\begin{abstract}
Summary
A clinical study was undertaken to determine the incidence of the dumping syndrome following partial gastrectomy and Roux-en-y gastrojejunostomy. Twenty-three patients were scored according to a clinical diagnostic index. There were no dumpers identified. A theoretical explanation for these findings is offered. A Roux-en-y conversion should be considered for patients with disabling dumping symptoms following gastric surgery.
\end{abstract}

\section{Introduction}

Reports on the incidence of the dumping syndrome following gastric surgery are notoriously variable, no doubt due largely to the lack of a precise definition for the syndrome. Visick (1948) claimed a figure of $16 \%$ in patients who had a Billroth II type gastrectomy. However, Goligher and Riley (1952) reported an overall incidence of $75 \%$ following subtotal gastrectomy, with no significant difference between the types of anastomosis, and with an incidence of disabling symptoms of 10 to $15 \%$.

Although the Roux-en-y gastrojejunal anastomosis (Fig. 1) has been advocated for a variety of postgastrectomy syndromes including dumping, there is little information regarding the overall incidence of dumping following this procedure. As it was our clinical impression that the incidence was very low, it was decided to review all our patients who had undergone partial gastrectomies with Roux-en-y anastomoses for benign disease.

\section{Method}

Records of surviving patients who had undergone a partial gastrectomy with Roux anastomosis as either a primary or revisional procedure were obtained. Only those whose pathology was benign were included.

Requests for reprints: Mr John O. Kilby, Gloucestershire Royal Hospital.

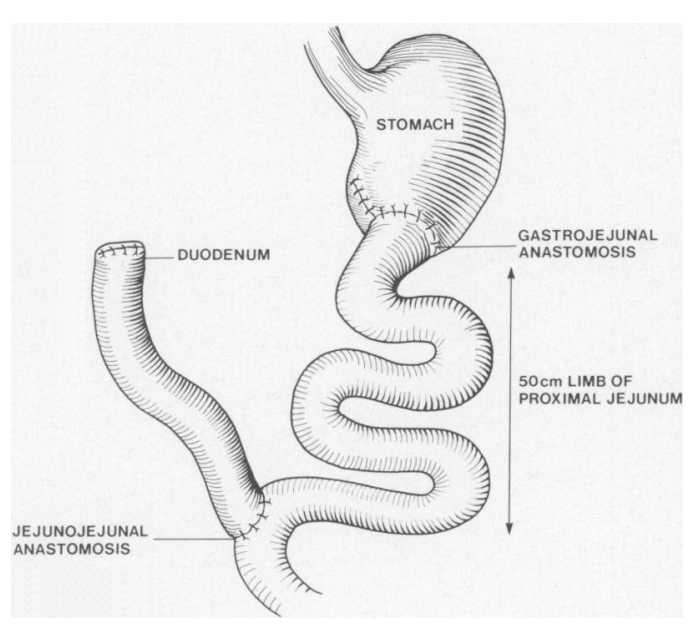

FIG. 1. The Roux-en-y gastrojejunal anastomosis.

Of the 25 patients on whom records were thus obtained, 23 were interviewed by one of us. On the basis of the interview all were classified according to a clinical diagnostic index described by Sigstad (1970) (Table 1). A level of +7 or above indicates dumping and +4 or below non-dumping.

\section{Results}

For 13 of the 23 patients the gastrojejunostomy with Roux anastomosis was the primary procedure. Seven patients had previously undergone a partial gastrectomy with or without vagotomy and hiatus hernia repair. Six had a Billroth II, one with a truncal vagotomy and one a Billroth I with vagotomy. Three patients had had previous hiatus hernia repairs, one with a pyloroplasty.

The indications for surgery were peptic oesophagitis with or without a stricture (12 cases) together with a duodenal ulcer diathesis (4 cases). Bile gastritis with 
TABLE 1. Clinical diagnostic index

Pre-shock, shock

'Almost fainting', syncope, unconsciousness

Desire to lie or sit down

Breathlessness, dyspnoea

Weakness, exhaustion

Sleepiness, drowsiness, yawning, apathy, falling asleep

Palpitation

Restlessness

Dizziness

Headache

Feeling of warmth, sweating, pallor, clammy skin

Nausea

Fullness in the abdomen, meteorismus

Borborygmia

Eructation

Vomiting

or without bile vomiting was the indication in four cases.

The final surgical state was as follows: nine subtotal gastrectomies, of whom two also had truncal vagotomies, and 14 antrectomies of whom eight also had truncal vagotomies and one a selective vagotomy.

There were no patients with a clinical score greater than 7 and only one in the equivocal range (Fig. 2).

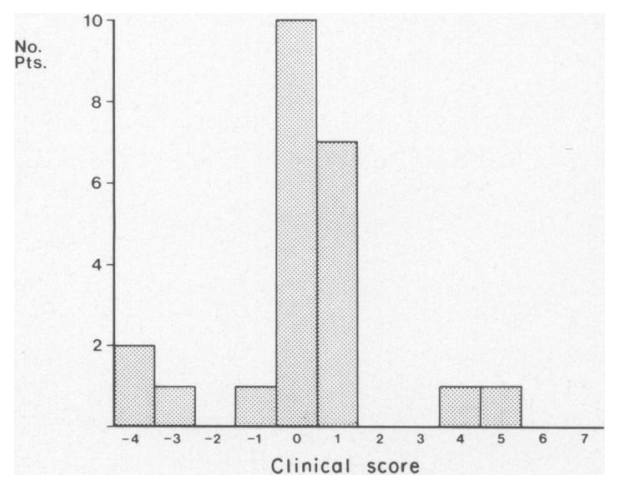

FIG. 2. Summary of clinical diagnostic scores.

\section{Discussion}

The diagnosis of the dumping syndrome is best made on the basis of the clinical manifestations of the vasomotor changes. Although raised levels of several gut peptides have been demonstrated in dumpersgastric inhibitory polypeptide (GIP) by McLoughlin, Buchanan and Alam (1979), neurotensin by Blackburn et al. (1978), enteroglucagon by Bloom, Royston and Thomson (1972) and vasoactive intestinal poly- peptide (VIP) by Sagor et al. (1980)-these measurements serve mainly as research tools. Sigstad (1970) has attempted to rationalize the clinical process by devising a clinical diagnostic index, and we have used this index in our study.

The mainstay in the management of dumping is dietary manipulation, including the addition of fibre. For the patient severely disabled by his symptoms numerous surgical remedies have been proffered. Conversion of a Billroth II anastomosis to a Billroth I is suggested in the belief that following the latter the incidence of dumping is less. However, success rates varying from 10 to $80 \%$ in series reported by Andreassen (1961), Capper and Welbourn (1955), Hinshaw, Stafford and Joergenson (1961) and Woodward and Bushkin (1976) reflect the need for a uniform definition for the syndrome. Similarly, conflicting reports by Fenger et al. (1972) and Cushieri (1977) demonstrate the difficulty in assessing the effectiveness of isoperistaltic versus antiperistaltic interposed jejunal loops, although the latter are probably more effective, but at the cost of a higher incidence of bile reflux related complications.

Nor is the efficacy of the Roux-en-y gastrojejunal anastomosis established. Wells and Johnston (1956) reported that their three patients who had Roux conversions for dumping symptoms were cured, whereas Conyers et al. (1960) only saw an improvement in seven out of 22 . However, the latter group admit that some of their failures may have been due to the loop being too short.

In this series none of the Roux-en-y anastomoses were done for the dumping syndrome, but we were impressed by the lack of vasomotor symptoms in these patients. There were no patients with a convincing dumping syndrome and only one with even equivocal symptoms. However, there were two other patients not included in the series who warrant mention. One, excluded because of our inability to interview him, had a Roux-en-y conversion for disabling dumping following selective vagotomy and pyloroplasty. Apart from minor dyspeptic problems he had been cured. On the other hand, one of our patients who underwent a Roux-en-y gastrojejunostomy for a gastrocolic fistula following gastrectomy for a lymphosarcoma (excluded from the series because of malignancy) complained of mild dumping symptoms after both operations.

On a theoretical basis these findings come as no surprise. Our current concept of the aetiology of the dumping syndrome is that sudden loading of the upper small intestine with the products of carbohydrate digestion produces an osmotic load resulting in a shift of fluid from the intravascular compartment and therefore hypovolaemia. The degree of hypovolaemia, however, does not necessarily correlate with the severity of the attack, nor does hypovolaemia 
account for some of the frequently encountered clinical findings such as facial flushing and peripheral vasodilation. The vasomotor symptoms would be better explained by the increased levels of the vasoactive peptides, VIP and neurotensin known to occur in this syndrome. GIP may also be involved by enhancing insulin secretion and producing reactive hypoglycaemia.

As digestion of 'digestible' carbohydrate is complete by the time a meal reaches the distal duodenum and subsequent absorption in the upper jejunum is very rapid, it is not surprising that the dumping provocation sequence can be avoided by bypassing the duodenum and upper jejunum. The length of the limb of the Roux-en-y must be generous; the ones in this series range from 30 to $50 \mathrm{~cm}$ (Fig. 1).

Although the place of the Roux-en-y anastomosis amongst the array of surgical procedures recommended for disabling dumping is contentious, its simplicity and relative freedom from dumping complications makes it worthy of consideration when revisional surgery is required.

\section{References}

ANDREASSEN, M. (1961) Surgical treatment of severe dumping syndrome. Acta chirurgica Scandinavica (Supplement) 283, 221.

BlackbuRN, A.M., Bloom, S.R., Ebeid, F.H., \& RalPhS, D.N.L. (1978) Neurotensin and the dumping syndrome. Gut, 19, A447.

Bloom, S.R. Royston, C.M.S. \& ThomSon, J.P.S. (1972) Enteroglucagon release in the dumping syndrome. Lancet, ii, 789.
CAPPER, W. \& Welbourn, R.B. (1955) Early postcibal symptoms following gastrectomy. Aetiological factors, treatment and prevention. British Journal of Surgery, 43, 24.

Conyers, J.H. Hall, R.A., Laing, D., Pulvertaft, C.N. \& WILLSON-PEPPER, J.K. (1960) The use and risks of the Roux-en-y anastomosis in the relief of post-gastrectomy symptoms. British Journal of Surgery, 47, 533.

CUSCHIERI, A. (1977) Isoperistaltic and antiperistaltic jejunal interposition for the dumping syndrome. Journal of the Royal College of Surgeons of Edinburgh, 22, 319.

Fenger, H.J., Gudmand-Hoyer, G.E., Kallehauge, H.E. \& ANDREASSEN, M. (1972) Clinical experience with isoperistaltic interposition of a jejunal segment for the incapacitating dumping syndrome. Annals of Surgery, 175, 274.

GOLIGHER, J.C. \& RILEY, T.R. (1952) Incidence and mechanism of the early dumping syndrome after gastrectomy. A clinical and radiological study. Lancet, i, 630.

Hinshaw, D.B., StafFord, C.E. \& Joergenson, E.J. (1961) Further observations on the surgical treatment of the dumping syndrome. American Journal of Surgery, 102, 38.

Mcloughlin, J.C., Buchanan, K.D. \& Alam, M.J. (1979) A glycoside-hydrolase inhibitor in treatment of dumping syndrome. Lancet, ii, 603.

SAGOR, G.R., Bryant, M.G., Ghatel, M.A., KIRK, R.M. \& Bloom, S.R. (1980) Release of VIP (vasoactive intestinal peptide) in dumping provocation. Gut, 21, A931.

SigSTAD, H. (1970) A clinical diagnostic index in the diagnosis of the dumping syndrome. Acta medica Scandinavica, 188, 479.

VISICK, A.H. (1948) A study of the failures after gastrectomy. Annals of the Royal College of Surgeons of England, 3, 266.

Wells, C. \& JohnSton, J.H. (1956) Revision to the Roux-en-y anastomosis for post-gastrectomy syndromes. Lancet, ii, 479.

WOODWARD, E.R. \& BUSHKIN, F.L. (1976) The early postprandial dumping syndrome: prevention and treatment. Major Problems in Clinical Surgery, 20, 14. 